\title{
LA REFLEXIÓN EN EL APRENDIZAJE DE LOS ESTUDIANTES DEL QUINTO AÑO DE SECUNDARIA DE LIMA METROPOLITANA
}

\author{
Luis M. Escurra Mayaute \\ Magister en Psicología Educativa
}

\begin{abstract}
RESUMEN
Se estudio el uso de la reflexión en el aprendizaje de los estudiantes del quinto año de secundaria de Lima Metropolitana. Los participantes correspondieron a una muestra probabilística estratificada de 942 alumnos pertenecientes de manera representativa a las diferentes USE. Los hallazgos del análisis psicométrico demostraron la validez de contenido, la validez de constructo y la confiabilidad de la escala de Reflexión en el Aprendizaje (ERA-2001).

Los resultados alcanzados indicaron que los puntajes de la reflexión en el Aprendizaje en los alumnos de quinto año de Lima Metropolitana presentaron una adecuada aproximación a la distribución normal. El análisis de la aplicación de la reflexión en el aprendizaje indicó que los alumnos de quinto año de secundaria de Lima Metropolitana no utilizan de forma significativa la reflexión en el aprendizaje.

Las comparaciones por tipo de colegio de procedencia indicaron que los alumnos de colegios particulares presentaron una mejor Integración, Monitoreo y Reflexión en el aprendizaje. El contraste por género, indicó que los varones presentan una mayor integración y reflexión en el aprendizaje que las mujeres. También se observó que no existieron diferencias estadísticas significativas según el grupo de edad y el lugar de procedencia.
\end{abstract}

Palabras Clave: Reflexión, Análisis Psicométrico, Confiabilidad, Validez, Normas.

\section{STRACT}

It was studied the reflection in learning in high school students of the city of Lima, Peru. The participants were a random sample of 942 students, belonging to the school districts (USE) of Lima.

The results of the psychometric analysis allows to state that content validity was obtained, the construct validity was calculated using the factorial confirmatory analysis, and the item-test relationship showed the reliability of the Reflection in Learning Profile (ERA-2001).

The results show that the scores in the Reflection in Learning Profile in high school students have a tendency to normal distribution. The analysis indicate that the students of high school do not use in a significant way the reflection in learning.

The comparison by kind of school indicate that the students of private schools show better integration, monitoring and reflection in learning. The comparison by gender points out that male students have a better integration and reflection on learning than their counterparts, this is, female students.

Also, it was observed no statistical differences neither considering age, nor residence place.

Key words: Reflection, psychometric analysis, reliability, validity, norms.

Miembros: Mg. Ana Delgado Vásquez - Ps. Rosario Quezada Murillo - Manuel Campos Roldan - Roger Elizalde Bravo - Víctor Montero López - Elio Córdova Huayanay.

Colaboradores: Julio Santos Islas - Gerardo Rivas Castro 


\section{LA REFLEXIÓN EN EL APRENDIZAJE}

La reflexión, según Boud, Keogh \& Walker (1988), es un proceso de pensamiento, el cual es activo y cuidadoso, pues constituye una actividad en que las personas recuerdan y evalúan sus experiencias, estos autores sugieren que la reflexión implica los siguientes aspectos:

a) Volver a las experiencias pasadas, recordando los eventos recientes.

b) Relacionarse con sus sentimientos, el cual tiene dos aspectos, el usar ayudas en base a los sentimientos previos y el remover los contenidos que lo obstruyen.

c) Experiencia evaluativa, que implica reexaminar las experiencias a la luz de los conocimientos, así como integrar este conocimiento en un nuevo marco conceptual de trabajo.

Para La Harpe y Radloff(2000) la reflexión implica una forma de aprendizaje a través del pensamiento activo que se sustenta sobre los aprendizajes pasados, de manera que permiten anticipar el futuro rendimiento y constituye un importante aspecto del aprendizaje autorregulado, de modo que los estudiantes que utilizan la reflexión en sus aprendizajes, presentan una mejor comprensión de los procesos que los regulan, por lo cual pueden ser capaces de ejercer un mejor control sobre su propio aprendizaje.

Como indica Mathison (1994), la noción de reflexión está basada en los conceptos de pensamiento reflexivo de Dewey y la concepción Piagetana Operatoria formal de los estadios del desarrollo cognitivo, es por ello que la reflexión incluye la habilidad para la autoobservación, la capacidad para analizar las experiencias pasadas, evaluar su significado y desarrollar un plan de acción basado en el análisis y la reflexión.

La reflexión está estrechamente unida a la metacognición (Biggs \& Moore, 1993), pues ésta incluye aprendizajes centrados en los procesos cognitivos, así como el uso de procedimientos que controlan y estimulan dichos procesos. La metacognición implica dos aspectos básicos de la cognición:

a. La atención en el conocimiento de la cognición que involucra variables relacionadas a las personas, estrategias de trabajo.

b. El control y regulación de la cognición, haciendo uso de la planificación, monitoreo, evaluación y el pensamiento adaptativo.

Angelo (1991) sugiere que los estudiantes para lograr alcanzar aprendizajes independientes necesitan aprender a ser autoreflexivos para comprender la forma cómo piensan y se desarrollan sus creencias. Además, indica que la reflexión es esencial para aprender y es el mecanismo central que facilita el desarrollo y el cambio cognitivo.

Para Ewell (1997), la reflexión es un proceso mental, el cual al ser aplicado al proceso de aprendizaje, puede motivar a los alumnos a usar su pensamiento crítico para examinar la información presentada, cuestionar su validez y sacar conclusiones basadas en sus ideas resultantes. Estos procesos permiten que los alumnos limiten las posibles soluciones y formulen eventualmente una conclusión. El resultado de éste proceso es una mejor comprensión del concepto. Como indica Ewell (1997, p.9): «sin la reflexión, el aprendizaje termina rápidamente, sin la reorganización del pensamiento que el aprendizaje profundo requiere". Las situaciones de aprendizaje efectivo requieren de un determinado tiempo para pensar. Los alumnos también pueden reflexionar acerca de lo que ellos utilizan cuando efectúan sus procesos de pensamiento, de manera que pueden determinar que estrategias 
funcionan mejor, de manera que pueden aplicar dicha información para realizar aprendizajes futuros. El proceso de reflexión implica los siguientes aspectos.

a. La Metacognición (Donovan, Bransford, \& Pellegrino. 1999, p.14). Este diálogo interno que los individuos desarrollan por su cuenta les ayuda a construir las habilidades de predecir los logros del aprendizaje y monitorear la comprensión.

b. La Transferencia de conocimientos (Donovan et al., 1999, p.6): Los alumnos pueden aplicar lo que han aprendido en un contexto determinado a un nuevo contexto.

a. El Razonamiento analógico (Donovan et al., 1999, p.13): Los alumnos pueden comparar y contrastar lo que conocen y les es familiar para tratar de encontrar significados $\mathrm{y}$ soluciones aplicables a un contexto en particular.

La reflexión según Ewell (1997), puede formar parte del aprendizaje autorregulado, el cual ocurre cuando el alumno ejecuta y mantiene conductas sistemáticamente orientadas hacia el cumplimiento de objetivos; en este caso, la adquisición de conocimientos implica actividades dirigidas por metas, que los estudiantes son capaces de poner en marcha, mantener y modificar. Para Schunk, (1990), una meta es lo que un individuo está intentando conseguir de forma consciente, y formular una meta implica establecerla, y modificarla si se considera necesario, la meta sería una representación cognitiva de lo que queremos que suceda (o de lo que deseamos evitar) en el futuro.

En el contexto del aprendizaje escolar, las metas pueden tener distintos orígenes: el alumno genera sus propias metas, adopta las propuestas por otros, desarrolla metas con otros, o recibe metas impuestas externamente (Karniol y Ross, 1996). El alumno no sólo debe asumir las distintas metas que se le plantean y seleccionarlas, sino que puede decidir qué es lo importante para él y formular sus propios objetivos de actuación. Para esto se requiere que el sujeto se conozca a sí mismo y que tenga unas expectativas realistas sobre lo que es capaz de llevar a cabo; también debe ser consciente de sus preferencias, intereses y valores, para lo que se necesita un cierto nivel de autoconciencia (Patalano y Seifert, 1997).

A partir de este autoconocimiento, el sujeto dispone de los instrumentos para seleccionar o plantear sus propias metas personales y significativas; asimismo, puede evaluar sus posibilidades de éxito o fracaso, generar expectativas de resultados y comprometerse a alcanzar sus metas. En esta fase, además del autoconocimiento, es fundamental la capacidad para reflexionar y evaluar la importancia y la relevancia de los objetivos elegidos, primer paso esencial para generar compromisos de actuación duraderos y sentimientos positivos hacia la consecución de las metas.

Como indican Vermunt (1995) y Justicia (1996), el aprendizaje autoregulado en los estudiantes en general, parece estar constituido por tres aspectos básicos:

a. Las estrategias metacognitivas de autoregulación, planificación, autocontrol y consciencia del propio estudio y sus efectos.

b. Las estrategias cognitivas, que constituyen procedimientos intencionales que permiten al estudiante tomar decisiones respecto a su estudio y rendimiento.

c. Las estrategias de Apoyo, que incluyen a la motivación, afecto y actitudes y que están relacionadas al control personal del esfuerzo, de la persistencia y a promover las condiciones que faciliten el estudio. 
El estudio de la reflexión, en la actualidad implica el desarrollo de tres grandes campos de investigación:

a. El estudio del rol que desempeña la reflexión en la transformación de las experiencias de los sujetos en aprendizajes, como es el caso de Boud, Keogh \& Walker (1988).

b. El estudio de las características de las estrategias de procesamiento del aprendizaje, según Richardson (1995), incluye a los siguientes enfoques:

b1. Enfoque profundo del aprendizaje, que tiende a producir elementos que representan un entendimiento más sustancial de las tareas de aprendizaje, en razón del desarrollo personal y la búsqueda del significado y la integración de un nuevo conocimiento en uno ya adquirido.

b2. Enfoque superficial del aprendizaje, que tiende a producir elementos que representan un bajo entendimiento y es de carácter reproductivo, debido a la memorización desorganizada de hechos y de conceptos. Para Gibbs (1992), la reflexión es una estrategia que favorece el enfoque profundo del aprendizaje, resaltando como sus principales componentes la conciencia de lo que se aprende y la conciencia de cómo se aprende.

c. El estudio de la metacognición del aprendizaje, especialmente en lo que se refiere al rol que desempeña la percepción de la autoeficacia en la regulación de las actividades metacognitivas como es el caso de Zirnmerman, Bandura y Martínez Pons (1992) quienes indicaron que la eficacia en la autoregulación del aprendizaje afecta las metas que se pueden establecer los estudiantes por sí mismos.

Existe una gran cantidad de trabajos empíricos sobre el aprendizaje autoregulado que se han realizado con escolares, notándose que la instrucción en la escuela tradicional se caracteriza por la existencia de un fuerte control externo en el cual el profesor asume la responsabilidad de regular el aprendizaje de sus alumnos (Vermunt, 1995).

Como principal antecedente del presente estudio, encontramos que Sobral (1998) construyó la escala de Reflexión en el Aprendizaje para evaluar la capacidad de aprendizaje autodirigido de los estudiantes universitarios de primeros ciclos, alcanzando resultados significativos.

En nuestro contexto no existen estudios similares y este concepto ha sido evaluado de forma diferente bajo el concepto de estilos de aprendizaje de Kolb, habiéndose desarrollado los estudios de Eisler (1989), Escurra (1991).

Es por todo lo antes expuesto, que podemos indicar que la Reflexión en el campo de la Psicología Educativa actual, constituye un aspecto muy importante en el análisis de los procesos de aprendizajes cognitivos, pues como mencionan Hammond y Collins (1991), la reflexión es una estrategia básica para desarrollar el aprendizaje autodirigido, motivo por el cual el propósito de la presente investigación fue estudiar el uso de la Reflexión en el aprendizaje de los alumnos de quinto año de secundaria de Lima Metropolitana.

\section{OBJETIVOS}

\section{a. Generales}

Estudiar el uso de la Reflexión en el aprendizaje de los alumnos de quinto año de secundaria de Lima Metropolitana 


\section{b. Específicos}

1. Adaptar la escala de Reflexión en el aprendizaje.

2. Evaluar la Confiabilidad de la escala de Reflexión en el aprendizaje.

3. Estudiar la Validez de Constructo de la escala de Reflexión en el aprendizaje.

4. Evaluar el uso de la Reflexión en el aprendizaje de los alumnos de quinto año de secundaria de Lima Metropolitana

5. Estudiar la existencia de diferencias significativas en la aplicación de la Reflexión en el aprendizaje de los alumnos de quinto de secundaria según el sexo.

6. Establecer la existencia de diferencias significativas en la aplicación de la Reflexión Activa en el aprendizaje de los alumnos de quinto de secundaria según el tipo de colegio al que pertenecen.

\section{MÉTODO}

La presente Investigación se realizó en dos momentos, en el primer momento se trabajo la escala de Reflexión en el aprendizaje, siguiendo a Kerlinger (2002), se trató de una investigación de Tipo Metodológico, en la medida que se trata de evaluar la Validez y Confiabilidad de la escala de Reflexión en el Aprendizaje.

La investigación también puede ser clasificada como aplicada, en la media que se orientó a la aplicación de manera práctica de los conocimientos teóricos de un determinado tema.

En el segundo momento se estudió la Reflexión en el aprendizaje, en los alumnos de quinto de secundaria de Lima Metropolitana se trata de una investigación Descriptiva (Sánchez y Reyes, 2002).

El diseño de la investigación en un primer momento correspondió al Correlacional en la medida que los resultados obtenidos en la medición del fenómeno fueron utilizados para analizar las características psicométricas del instrumento utilizado (Alarcón, 1991; Hernández, Fernández y Baptista, 1998; Sánchez y Reyes, 2fl02).

En el momento que se desarrolló la investigación Descriptiva sobre el uso de la reflexión en el aprendizaje en los alumnos de quinto de secundaria de Lima Metropolitana, se utilizó un diseño de tipo Descriptivo Comparativo en la medida que los resultados obtenidos en la medición del fenómeno fueron comparados de acuerdo al tipo de colegio de procedencia y el sexo (Hernández, Fernández y Baptista, 1998).

\section{Participantes}

El universo de investigación estuvo conformado por 79,128 alumnos matriculados en 5to año de secundaria en Lima metropolitana, de acuerdo a las estadísticas reportadas por el Ministerio de Educación.

El tamaño de la muestra de investigación fue establecido a través del programa informático SOTAM, el cual determinó que con un margen de error del $5 \%$ Y un nivel de confianza del $99.9 \%$, le correspondió un tamaño de muestra de 942 casos.

Para la obtención de la muestra se aplicará un procedimiento de muestreo probabilístico Estratificado, clasificando a los alumnos de acuerdo al sexo, tipo de colegio de procedencia y la USE a la que pertenecen. 


\section{Composición de la muestra}

La muestra estuvo constituida por 942 alumnos (ver tabla No.1), con edades que fluctuaban entre los 14 y los 18 años, siendo la mayor cantidad la que correspondió a los que tuvieron 16 años (46,20\%). También se observó que el 51,90\% fueron mujeres y el $48,10 \%$ fueron varones.

Respecto al colegio de procedencia existieron más alumnos pertenecientes a colegios estatales $(73,90 \%)$ que a colegios particulares $(26,10 \%)$. La mayoría de alumnos eran de Lima $(71,40 \%)$. Con relación al nivel socioeconómico se observó que el $85,00 \%$ eran del nivel medio bajo, el 10,60 del nivel bajo y el 4,40 del nivel medio. También se encontró que la gran mayoría vivía con su familia $(97,80 \%)$.

\section{Instrumento}

En la presente investigación se aplicó la Escala de Reflexión en el Aprendizaje (ERA2001) desarrollada por Sobral (1998), tomando como base a diferentes instrumentos psicológicos, llegando a elaborar su escala, la cual estuvo originalmente conformada por 10 ítems, pero que para nuestro estudio se consideraron sólo 8 de ellas, debido al tipo de contexto educativo estudiado.

Tabla No. 1

Composición general de la muestra de alumnos evaluados

\begin{tabular}{lcc}
\hline \multicolumn{1}{c}{ Variable } & F. & \% \\
\hline Edad & & \\
14 años & 24 & 2,50 \\
15 años & 206 & 21,90 \\
16 años & 435 & 46,20 \\
17 años & 218 & 2,10 \\
18 años & 59 & 6,30 \\
Sexo & & \\
Varón & 453 & 48,10 \\
Mujer & 489 & 51,90 \\
Tipo de Colegio & & \\
Estatal & 696 & 73,90 \\
Particular & 246 & 26,10 \\
Lugar de procedencia & & \\
Lima & 673 & 71,40 \\
Callao & 157 & 16,70 \\
Provincia & 112 & 11,90 \\
Nivel Socio-Económico & & \\
Medio & 41 & 4,40 \\
Medio Bajo & 801 & 85,00 \\
Bajo & 100 & 10,60 \\
Con quien Vive & & \\
Sólo & 7 & 0,70 \\
Con su familia & 921 & 97,80 \\
Otro & 14 & 1,50 \\
\hline
\end{tabular}

$\mathrm{N}=942$ 
La escala aplicada en el presente estuvo constituida por 8 afirmaciones acerca de diferentes aspectos asociados a la aplicación de la reflexión en el aprendizaje escolar y que miden los siguientes aspectos relacionados a la reflexión en el aprendizaje:

a. Integración en el Aprendizaje, que alude al uso de la reflexión como elemento integrante del proceso de aprendizaje.

b. Monitoreo del Aprendizaje, que se refiere al uso de la reflexión como procedimiento para guiar y controlar el aprendizaje.

En la escala, todos los enunciados se califican sobre la base de un sistema tipo likert con 7 puntos de calificación, los cuales incluyen las siguientes opciones :

- Nada(l)

- Casi nada (2)

- Ligeramente(3)

- Un poco (4)

- Bastante (5)

- Mucho (6)

- Totalmente (7).

El instrumento puede ser aplicado tanto de forma individual como colectiva y está diseñado para ser administrado a grupos de adolescentes por el tipo de afirmaciones que contiene. El tiempo de duración de la aplicación del instrumento es de aproximadamente entre 5 y 10 minutos.

\section{Validez y Confiabilidad de la Escala original}

En su estudio Sobral (1998), encontró que la escala presentaba un adecuado análisis de ítems, encontrando que las correlaciones ítem test corregidas fluctuaban entre 0.40 y 0.65 y también reportó que se alcanzó una confiabilidad a través del coeficiente Alfa de Cronbach de $0.80 \mathrm{y}$, además, que la prueba presentó validez predictiva y validez de constructo.

Para el desarrollo del presente estudio se realizó la adaptación psicométrica del instrumento, motivo por el cual se desarrolló la traducción de la escala de la versión portuguesa al castellano, revisándose la adecuación lingüística de los ítems y el respectivo análisis psicométrico.

\section{Análisis de los datos}

El análisis estadístico fue realizado tomando en cuenta las recomendaciones propuestas por Siegel y Castellan (1995), el cual abarcó las siguientes etapas:

En primer lugar, se efectuó el análisis psicométrico de la escala, que implicó la ejecución del análisis de ítems y la confiabilidad a través del coeficiente Alfa de Cronbach.

En segundo lugar se estudió la validez que fue evaluada a través de la Validez de Contenido por Criterio de Jueces y la Validez de Constructo por medio del Análisis factorial Confirmatorio.

En tercer lugar, para el análisis comparativo de los resultados se cálculo en primer lugar la prueba de bondad de ajuste a la curva normal de Kolmogorov-Smirnov, como los resultados indicaron que los puntajes presentaron adecuadas aproximaciones a la curva normal, se optó por utilizar estadísticas Paramétricas, así, para la comparación por Colegio de procedencia y por Género, se aplicó la prueba t de student para grupos independientes. Para la comparación por grupo de edad y Lugar de Procedencia, se empleó el Análisis de 
Varianza de un factor y la prueba de comparación post-hoc de Scheffé.

\section{RESULTADOS}

\section{Análisis Psicométrico}

Los resultados del Análisis de ítems efectuados por medio del coeficiente de correlación ítem-test corregido, presentados en la Tabla No. 2, permiten apreciar que se alcanzan correlaciones mayores que 0.45 , los cuales superan el criterio de 0.20 propuesto por Kline (1995), por lo cual podemos indicar que todos los ítems son relevantes para la escala.

El análisis de la confiabilidad por consistencia interna, calculado a través del coeficiente Alfa de Cronbach, asciende a 0.84 , por lo que se puede concluir que la escala de Reflexión en el Aprendizaje permite obtener puntajes confiables.

Tabla No. 2

Análisis de ítems y con fiabilidad de la escala de Reflexión en el Aprendizaje

\begin{tabular}{cccc}
\hline Items & M & D.E. & .ritc \\
\hline Item 1 & 3,64 & 1,23 & $0,54^{*}$ \\
Item2 & 4,01 & 1,42 & $0,46^{*}$ \\
Item3 & 4,63 & 1,41 & $0,49^{*}$ \\
Item4 & 3,91 & 1,43 & $0,54^{*}$ \\
Item5 & 3,32 & 1,37 & $0,63 *$ \\
Item6 & 2,93 & 1,38 & $0,58^{*}$ \\
Item7 & 3,22 & 1,40 & $0,60^{*}$ \\
Item 8 & 3,14 & 1,41 & $0,53 *$ \\
\hline
\end{tabular}

Coeficiente Alta de Cronbach $=0.84$

* $\mathrm{p}<.05$

$\mathrm{N}=942$

Tabla $\mathbf{N}^{\mathbf{0}} 3$

Validez de contenido por Criterio de Jueces de la Escala de Reflexión en el Aprendizaje

\begin{tabular}{cc}
\hline Item & V Aiken \\
\hline $\mathbf{1}$ & $0,90^{*}$ \\
$\mathbf{2}$ & $0,90^{*}$ \\
$\mathbf{3}$ & $0,90^{*}$ \\
$\mathbf{4}$ & $0,90^{*}$ \\
$\mathbf{5}$ & $0,90^{*}$ \\
$\mathbf{6}$ & $0,98^{*}$ \\
$\mathbf{7}$ & $0,90^{*}$ \\
$\mathbf{8}$ & $0,90^{*}$ \\
\hline
\end{tabular}

$* \mathrm{p}<.05$

Jueces $=10$

El análisis psicométrico de la validez de contenido realizado a través del procedimiento de Criterio de Jueces, desarrollado con la participación de 10 evaluado res independientes quienes analizaron si los ítems eran representativos del dominio de contenido que se quería estudiar. Los resultados presentados en la Tabla No. 3, permite apreciar que todos los ítems alcanzaron un coeficiente $\mathrm{V}$ de Aiken igual a 0.90 el cual es significativo a $\mathrm{p}<.05$. Este 
hallazgo nos permite concluir que la Escala de reflexión en el Aprendizaje presenta Validez de Contenido.

El estudio de la Validez de Constructo efectuado a través del Análisis Factorial Confirmatorio, aplicando el programa Amos 4.0, se analiza la existencia de una dimensión que evalúa la escala (ver Tabla No. 9 y Grafico No.1), se observa que el modelo propuesto de 2 factores presenta en el test de Bondad de Ajuste Chi-Cuadrado Mínimo (Cmin) un valor de 19,58, el cual con 17 grados de libertad alcanza una probabllidad de 0,296, lo que nos indica que el modelo es adecuado, notándose, además, que este resultado es superior al modelo Independiente, lo cual es corroborado con los estadísticos de análisis de residuales (RMR) y los análisis complementarios de bondad de Ajuste como son el GFI y el AGFI. Estos Hallazgos nos indican que la escala de Reflexión en el Aprendizaje presenta validez de Constructo.

Tabla No. 4

Validez de Constructo a través del Análisis Factorial

Confirmatorio de la escala de Reflexión en el

\begin{tabular}{lcc}
\multicolumn{1}{c}{ Datos } & $\begin{array}{c}\text { Modelo de 2 } \\
\text { Factores }\end{array}$ & $\begin{array}{c}\text { Modelo } \\
\text { Independiente }\end{array}$ \\
\hline Parámetros & 27 & 44 \\
Cmin & 19.58 & 17386,68 \\
G.L. & 27 & 36 \\
P & 0,296 & 0,000 \\
Cmin/G.L. & 1,152 & 482,963 \\
RMR & 0.013 & 0,716 \\
GFI & 0,999 & 0,503 \\
AGFI & 0,998 & 0,361 \\
\hline
\end{tabular}

$\mathrm{n}=942$

\section{Análisis Descriptivo}

Los resultados del análisis de la bondad de ajuste a la curva normal, realizado a través de la prueba de Kolmogorov-Smimov (ver Tabla No. 5 y Gráfico No. 2), indican que tanto en los factores 1 (Integración en el Aprendizaje) y 2 (Monitoreo del Aprendizaje) y en el Puntaje total, se obtienen estadísticos que no son significativos, por lo que se puede concluir que la distribución de las variables analizadas se aproximan adecuadamente a la distribución normal. Es Por ello que se utilizaran contrastes estadísticos Paramétricos en el análisis de los datos de la investigación.

\section{Tabla $\mathbf{N}^{\circ} 5$}

Bondad de Ajuste a la Curva Normal de Kolmogorov-Smirnov de las Escalas de Reflexión en el Aprendizaje

\begin{tabular}{|c|c|c|c|}
\hline VARIABLE & MEDIA & D.E & K-S Z \\
\hline Integración en el Aprendizaje & 16.19 & 3.93 & 0.89 \\
\hline Monitoreo del Aprendizaje & 12.61 & 4.27 & 0.75 \\
\hline Reflexión en el Aprendizaje & 28.81 & 7.41 & 0.7 \\
\hline
\end{tabular}

$\mathrm{N}=942$ 


\section{Análisis Inferencial}

Con relación a los resultados del análisis de la aplicación de la reflexión en el Aprendizaje de los alumnos de 5to. año de secundaria, en primer lugar se trato de establecer el nivel con el que se presentaba de forma general, para ello se utilizó como punto de comparación los resultados alcanzados por Sobral (1998) pero ponderados a los valores de los 8 ítems que conforman la escala utilizada en el presente estudio. En la Tabla No. 6 se aprecia que existen diferencias estadísticas significativas $(Z=-38,09 ; \mathrm{p}<.001)$, notándose que el valor observado en los alumnos es inferior al valor esperado.

Tabla $\mathbf{N}^{\circ} 6$

Prueba $\mathrm{Z}$ de comparación con respecto a una media teórica de los múltiples de la Reflexión en el Aprendizaje

\begin{tabular}{cccc}
\hline Variable & Media & D.E & $\mathbf{Z}$ \\
\hline $\begin{array}{c}\text { Reflexión en el } \\
\text { Aprendizaje }\end{array}$ & 28.81 & 7.41 & $-38.09 * * *$ \\
***p<,001 & & &
\end{tabular}

Media teórica $=38$

$\mathrm{N}=942$

\section{Grafico No 1}

Modelo de dos factores de la escala

de reflexión en el Aprendizaje
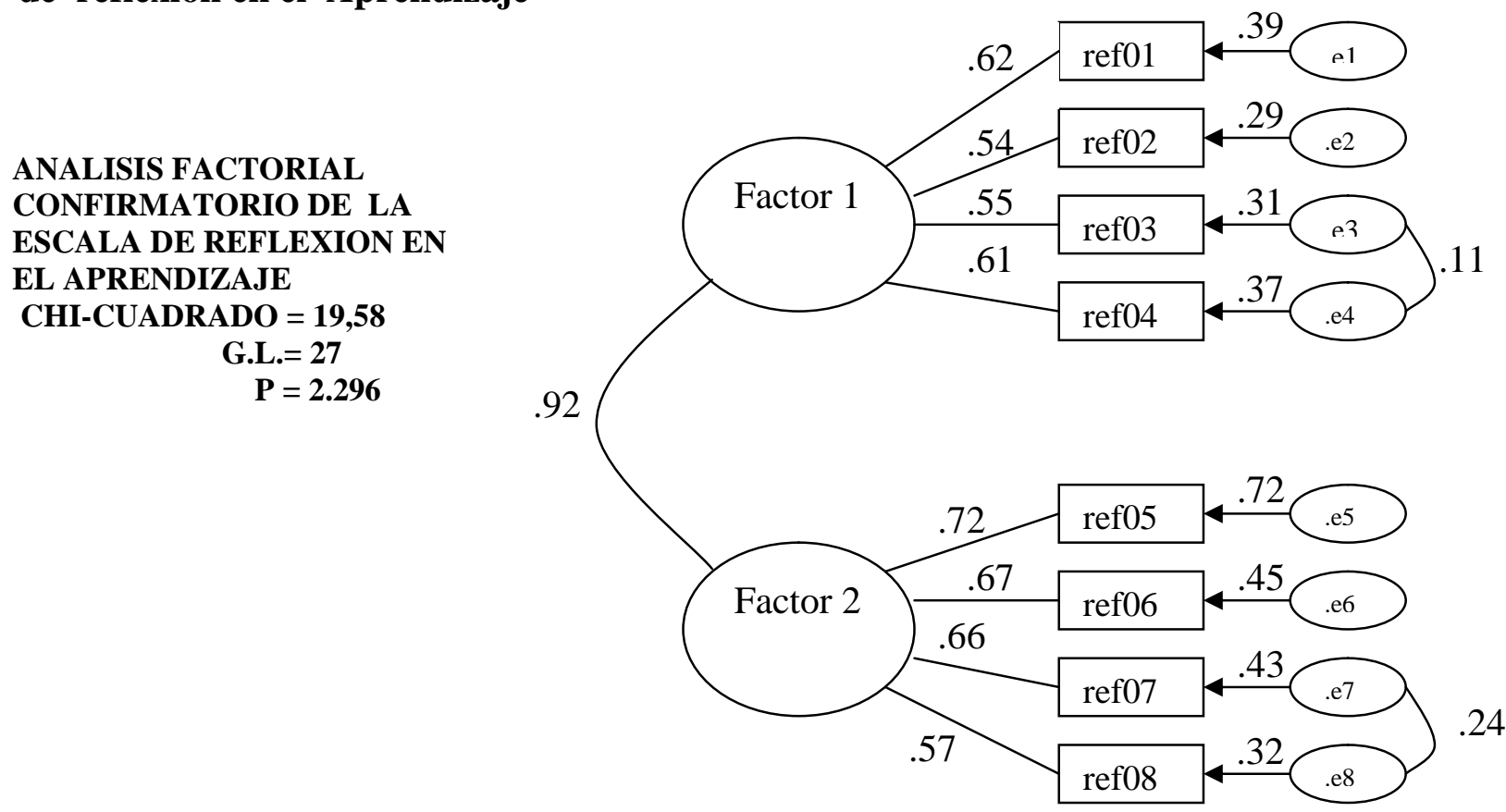


\begin{tabular}{|c|c|c|c|}
\hline \multicolumn{4}{|c|}{$\begin{array}{c}\text { Gráfico } \mathrm{N}^{\circ} 2 \\
\text { Análisis de Tallos y Hojas de la Escala de Reflexión en el Aprendizaje }\end{array}$} \\
\hline Frecuencia & Tallo & $\&$ & Hojas \\
\hline 9.00 & 1 & * & 1 \\
\hline 9.00 & 1 & $\mathrm{t}$ & 223 \\
\hline 16.00 & 1 & $\mathrm{f}$ & 444555 \\
\hline 24.00 & 1 & $\mathrm{~s}$ & 66667777 \\
\hline 33.00 & 1 & . & 88888999999 \\
\hline 51.00 & 2 & * & 00000001111111111 \\
\hline 83.00 & 2 & $\mathrm{t}$ & 222222222222223333333333333 \\
\hline 80.00 & 2 & $\mathrm{f}$ & 44444444444455555555555555 \\
\hline 108.00 & 2 & $\mathrm{~s}$ & 66666666666667777777777777777777777777 \\
\hline 111.00 & 2 & . & 888888888888888888889999999999999999999 \\
\hline 89.00 & 3 & $*$ & 000000000000000111111111111111 \\
\hline 76.00 & 3 & $\mathrm{t}$ & 22222222222222333333333333 \\
\hline 85.00 & 3 & $\mathrm{f}$ & 44444444444444455555555555555 \\
\hline 69.00 & 3 & $\mathrm{~s}$ & 66666666666677777777777 \\
\hline 43.00 & 3 & . & 88888888999999 \\
\hline 16.00 & 4 & $*$ & 00011 \\
\hline 10.00 & 4 & $\mathrm{t}$ & 233 \\
\hline 10.00 & 4 & $\mathrm{f}$ & 4555 \\
\hline 10.00 & 4 & $\mathrm{~s}$ & $666 \&$ \\
\hline 1.00 & 4 & . & $\&$ \\
\hline
\end{tabular}

La comparación de los alumnos según el tipo de gestión del colegio (ver Tabla No, 7), permite observar que existen diferencias estadísticas significativas tanto en el Factor 1, el Factor 2 y el Puntaje Total, observándose en todos los casos que existen diferencias estadísticas significativas, notándose que los alumnos procedentes de los colegios particulares presentan mayores puntajes que los alumnos de los colegios estatales.

\section{Tabla $\mathbf{N}^{0} 7$}

Prueba $Z$ de comparación de medias de los puntajes de la Escala de reflexión en el Aprendizaje según tipo de Colegio

\begin{tabular}{cccccc}
\hline \multirow{2}{*}{ Variable } & \multicolumn{2}{c}{$\begin{array}{c}\text { Estatal } \\
\text { N=696 }\end{array}$} & \multicolumn{2}{c}{$\begin{array}{c}\text { Particular } \\
\text { N = 246 }\end{array}$} & \multirow{2}{*}{ Z } \\
\cline { 2 - 5 } & $\mathbf{M}$ & $\mathbf{D . E .}$ & $\mathbf{M}$ & $\mathbf{D . E .}$ & \\
\cline { 2 - 5 } Integración en el Aprendizaje & 16.02 & 3.89 & 16.67 & 3.99 & -2.29 \\
Monitoreo del Aprendizaje & 12.39 & 4.23 & 13.25 & 4.32 & -2.73 \\
Reflexión en el Aprendizaje & 28.4 & 7.33 & 29.94 & 7.52 & -2.79 \\
\hline
\end{tabular}

$* \mathbf{p}<, 05 * * \mathbf{p}<, 01$

En relación a la comparación por género de la escala de Reflexión en el Aprendizaje, en la Tabla No. 8, se encuentra que existen diferencias estadísticas significativas en la Integración en el Aprendizaje $(Z=3,05 ; p<, 05)$, y la escala total ( $Z$ 
$=2,49 ; \mathrm{P}<, 05)$, encontrándose que en ambos casos los varones alcanzan valores más elevados que las mujeres.

\section{Tabla $\mathbf{N}^{\circ} 8$}

Prueba $\mathrm{Z}$ de comparación de medias de los puntajes de las variables estudiadas por Género

\begin{tabular}{|c|c|c|c|c|c|}
\hline \multirow[t]{2}{*}{ Variable } & \multicolumn{2}{|c|}{$\begin{array}{c}\text { Estatal } \\
N=453\end{array}$} & \multicolumn{2}{|c|}{$\begin{array}{c}\text { Particular } \\
N=489\end{array}$} & \multirow[t]{2}{*}{$\mathrm{Z}$} \\
\hline & $\mathbf{M}$ & D.E & M & D.E & \\
\hline Integración en el Aprendizaje & 16.00 & 3.99 & 15.82 & 3.83 & -305 \\
\hline Monitoreo del Aprendizaje & 12.83 & 4.28 & 4.28 & 12.41 & -152 \\
\hline Reflexión en el Aprendizaje & 29.43 & 7.50 & 7.50 & 28.23 & -249 \\
\hline
\end{tabular}

$* \mathbf{p}<, 05 * * \mathbf{p}<, 01$

Respecto a la comparación de la Escala de Reflexión en el Aprendizaje según la edad cronológica de los estudiantes, en la Tabla No. 9, se puede apreciar que no existen diferencias estadísticas significativas en ninguno de los aspectos evaluados por la escala de Reflexión en el Aprendizaje.

Tabla $\mathbf{N}^{0} 9$

Análisis de varianza de un Factor de la Escala de Reflexión del aprendizaje por Edad Cronológica

\begin{tabular}{ccccccc}
\hline & \multicolumn{7}{c}{ Edad Cronológica } & \\
\cline { 2 - 6 } Variable & $\mathbf{1 4}$ & $\mathbf{1 5}$ & $\mathbf{1 6}$ & $\mathbf{1 7}$ & $\mathbf{1 8}$ & $\mathbf{f}$ \\
& $\mathbf{N = 2 4}$ & $\mathbf{N = 2 0 6}$ & $\mathbf{N = 4 3 5}$ & $\mathbf{N}=\mathbf{2 1 9}$ & $\mathbf{N = 5 9}$ & \\
& $\mathbf{M}$ & $\mathbf{M}$ & $\mathbf{M}$ & $\mathbf{M}$ & $\mathbf{M}$ & \\
\hline Integración en el Aprendizaje & 15.79 & 16.45 & 16.31 & 15.91 & 15.64 & 0.96 \\
Monitoreo del Aprendizaje & 11.75 & 13.46 & 12.32 & 12.40 & 13.42 & 1.5 \\
Reflexión en el Aprendizaje & 27.54 & 29.91 & 28.63 & 28.18 & 29.07 & 1.79 \\
\hline
\end{tabular}

$\mathrm{GL}_{1}=4 \mathrm{GL}_{2}=937$

En la comparación de la Escala de Reflexión en el Aprendizaje de acuerdo al lugar de procedencia ( ver Tabla No. 10), los resultados indican que no existen diferencias estadísticas significativas en los valores obtenidos.

Tabla $\mathbf{N}^{\circ} \mathbf{1 0}$

Prueba $\mathrm{Z}$ de comparación de medias de los puntajes de las variables estudiadas por Lugar de Procedencia

\begin{tabular}{ccccc}
\hline & \multicolumn{3}{c}{ Lugar de Procedencia } & \multirow{2}{*}{ Variable } \\
\cline { 2 - 4 } & Lima & Callao & Provincia & \\
\hline & $\mathbf{N = 6 7 2}$ & $\mathbf{N}=\mathbf{1 5 7}$ & $\mathbf{N}=\mathbf{1 1 2}$ & \\
& $\mathbf{M}$ & $\mathbf{M}$ & $\mathbf{M}$ & \\
\hline Integración en el Aprendizaje & 16.29 & 16.00 & 15.90 & 0.69 \\
Monitoreo del Aprendizaje & 12.62 & 13.11 & 11.88 & 2.77 \\
Reflexión en el Aprendizaje & 28.91 & 29.11 & 27.76 & 1.28 \\
\hline
\end{tabular}

$\mathrm{GL}_{1}=2 \mathrm{GL}_{2}=939$ 


\section{DISCUSIÓN}

Los resultados observados nos indican que los ítems de la escala de Reflexión en el Aprendizaje (ERA-2001), alcanzaron Coeficientes de correlaciones ítem-test significativas, así como valores de Confiabilidad adecuados, estos resultados concuerdan con los hallazgos de Sobral (1998), en sus estudios con estudiantes Brasileños, lo cual demostraría la factibilidad de contar con un instrumento de medición válido y confiable, que puede ser utilizado en nuestro medio.

La propuesta de Sobral (1998), sobre la estructura de la Reflexión en el Aprendizaje, que fue estudiada a través de la validez de constructo, permitió comprobar de acuerdo con los resultados del Análisis Factorial Confirmatorio, la existencia de los dos factores propuestos, la Integración en el Aprendizaje y el Monitoreo del Aprendizaje en nuestro contexto. Estos hallazgos son concordantes con la propuesta de Marín (1986), quien resalta la necesidad de estudiar los aspectos psicométricos como son la validez y confiabilidad de toda prueba psicológica que provenga de otros contextos socio-culturales.

Con relación a los análisis de la aplicación de la reflexión en el aprendizaje que realizan los alumnos de 5to. año de secundaria de Lima metropolitana, encontramos que los alumnos no aplican de forma consistente sus procesos de reflexión para desarrollar su aprendizaje, lo cual podría deberse a lo que indica Vermunt (1995), quien propone que en los ámbitos educativos es el profesor quien asume la responsabilidad de regular el aprendizaje de los alumnos, y además, estaría en concordancia con lo propuesto por Angelo (1991) quien índica que sólo los alumnos que llegan a poder aplicar la reflexión pueden lograr mejores rendimientos escolares.

La comparación de los alumnos según el tipo de gestión del colegio, permitió apreciar que en todos los casos existen diferencias estadísticas significativas, a favor de aquellos que proceden de colegios particulares respecto a los alumnos de colegios estatales, este resultado refleja el impacto que produce el tipo de enseñanza que es impartida en los colegios y que a su vez reflejaría de forma clara las diferencias en la calidad de educación que se imparte en nuestro medio.

Respecto a la comparación por género de la escala de Reflexión en el Aprendizaje, se ha encontrado que los varones presentan una mayor Integración y Reflexión en el Aprendizaje notándose que en ambos casos los varones alcanzan valores más elevados que las mujeres, este resultado podría deberse a las diferencias en los procesos de socialización, que reciben los varones y las mujeres en nuestro contexto, de manera que reciben influencias diferenciales sobre el desarrollo de las diferentes estrategias y procesos cognitivos.

Con relación a la no existencia de diferencias significativas tanto por grupo de edad, así como por el lugar de procedencia, estos hallazgos ponen en relieve que la Reflexión en el Aprendizaje es un proceso cognitivo que por encima de dichas variables sociodemográficas es más influenciable por los aspectos contextuales de la enseñanza escolarizada.

En conclusión los hallazgos observados justifican la necesidad de implementar programas que incentiven el desarrollo cognitivo de la reflexión en los alumnos de quinto de secundaria de manera que participen de forma activa en su aprendizaje, asumiendo la responsabilidad que les corresponde. 


\section{CONCLUSIONES}

Los resultados de la investigación indican que:

1. La Escala de Reflexión en el aprendizaje (ERA-2001), presenta validez de Contenido y Validez de Constructo.

2. La Escala de Reflexión en el aprendizaje presenta confiabilidad por consistencia interna.

3. Los puntajes de la reflexión en el Aprendizaje en los alumnos de quinto año presentan una distribución Normal.

4. Los alumnos de quinto año de secundaria de Lima Metropolitana no utilizan de forma significativa la reflexión en el aprendizaje.

5. Los alumnos de los colegios particulares aplican más la reflexión en el aprendizaje que los alumnos provenientes de los colegios estatales

6. Los varones utilizan más la reflexión en el aprendizaje, la Integración y el Monitoreo del Aprendizaje que las mujeres.

7. No existen diferencias significativas en el uso de la reflexión según la edad de los alumnos.

8. No existen diferencias significativas en el uso de la reflexión según el lugar de procedencia de los alumnos.

\section{REFERENCIAS}

Alarcón, R. (1991). Métodos y diseños de Investigación del Comportamiento. Lima: U.P.C.H., Fondo Editorial.

Anastasi, A. Urbina, S. (1998). Test Psicológicos. México: Editorial Prentice Hall. Angelo, T. A. (1991). Ten easy pieces: Assessing higher learning in four dimensions. New Directions for Teaching and Learning, 46,pp.17-31

Biggs, J. B. \& Moore, P. J. (1993). The process of learning. Sydney: Prentice Hall.

Boud, D., Keogh, R. \& Walker, D. (1988). Reflection: turning experience into learning. London: Kogan Page.

Brown, F. (1980). Principios de la Medición en Psicología y Educación. México: Edit. El Manual Moderno.

Donovan, S., Bransford, J., \& Pellegrino, J. (Eds). (1999). How people learn: Bridging research and practice. National Academy of Sciences [On-line]. Available: http:// bob.nap. edu/html/howpeople2/.

Eisler S., H. (1989). Adaptación del Inventario de Estilos de Aprendizaje de Kolb. Facultad de Humanidades. PUCP

Escurra M.,L.M. (1991). Adaptación del Inventario de Estilos de Aprendizaje de Kolb en estudiantes de Psicología pertenecientes diferentes Universidades de Lima Metropolitana Facultad de Psicología. Tesis para optar el título de Psicólogo. UNMSM.

Ewell, P. T. (1997). Organizing for learning: A point of entry. Draft prepared for discussion at the 1997 AAHE Summer Academy at Snowbird. National Center for Higher Education Management Systems (NCHEMS). Available:

http://www.intime.uni.edu/model/Spanish_Model/ learning/learn_summary.htm1.

Gardner, H. (1993). Estructuras de la mente: La teoría de las Múltiples inteligencias. México: Fondo de Cultura Económica. Gibbs, G. (1992). Improving the quality of student learning. Bristol: technical and Educational Services. 
Hammond, M. \& Collins, R. (1991). Self directed learning in practice. London: Kogan Page.

Hemández, Femández, Baptista. (1998). Metodología de la investigación. México: Mc. Graw-Hill.

Justicia, F. (1996). Metacognición y Curriculum. En Beltrán, J. y Genovard, C. (eds,.) Psicología de la Instrucción I. variables y procesos. (pp. 359-382).

Karniol, R., y Ross, M. (1996). The motivational impact of temporal focus: thinking about the future and the past. Annual Review of Psychology, 47,593-620.

Kerlinger, F. y Lee, H. (2002). Investigación del comportamiento: Métodos de Investigación en las Ciencias Sociales. México: Mac Graw-Hill.

La Harpe, B. y Radloff, A. (2000) Do first students reflect on their learning? Why they should and how they can. Camberra: Intemational Press.

Mathison, S. (1994). Critical reflection on classroom practice: Teaching as an investigative activity. In E. W. Ross (ed.), Reflective practice in social studies (pp.59-

68). Washington D. C.: NCSC.

Marín, G. (1986). Consideraciones metodológicas básicas para conducir investigaciones Psicológicas en América Latina. Acta Psiquiátrica y Psicológica de América de Latina, Vol. 32, Pgs. 183-192.

Muñiz, J. (1996). Psicometría. Madrid: Ed. Universitas S. A.

Patalano, A. L. y Seifert, C. M. (1997). Oportunistic planning: being reminded of pending goals. Cognitive Psychology, 34,1-36.

Richardson, J. T. E. (1995),Using questionnaries to evaluate student learning. In G. Gibbs (Org.), Improving student larning through assesment and evaluation. Oxford: Oxford Centre for Staff Development.

Sánchez, C. H. y Reyes, M. C. (2002). Metodología y Diseños en la Investigación Científica: Aplicadas a la Psicología, Educación y Ciencias Sociales. Lima: U. R P.

Schunk, D. H. (1990). Goal setting and self-efficacy during self-regulated learning. Educational Psychologist, 25(1), 71-86.

Schutz, P. A. (1994). Goals as the transactive point between motivation and cognition. En P. R. Pintrich, D. R. Brown y C. E. Weinstein, Student motivation, cognition and learning. Essays in honor of Wilbert J. McKeachie. Hillsdale, NJ: Erlbaum.

Siegel, S. y Castellan N. (1995). Estadistica No paramétrica: Aplicada a las ciencias de la conducta. México: Editorial Trillas.

Sobral D. T. (1998). Desenvolvimiento e validacao de Escala de Reflexao na Aprendizagem. Psicología: Teoria e Pesquisa. Maio - Ago, Vol 14. N 2 pp. 173-177. Vermunt, J. D. (1995). Process-oriented instruction in learning and thinking strategies. European Journal of Psychology of Education. X (4). Pp. 325-349.

Zimmerman, B. J., Bandura, A. \& Martínez Pons, M. (1992). Self-motivation for academic attainment: The role of self-efficacy beliefs and personal goal setting. American Educational Research Journal, 29, pp. 663-676. 


\begin{tabular}{|c|c|c|c|c|}
\hline \multicolumn{5}{|c|}{ ANEXO } \\
\hline \multicolumn{5}{|c|}{ Tabla $N^{0} 11$} \\
\hline \multicolumn{5}{|c|}{ Baremo de la Escala de Aprendizaje } \\
\hline $\begin{array}{c}\text { Rango } \\
\text { Percentil }\end{array}$ & Factor 1 & Factor 2 & Escala Total & $\begin{array}{c}\text { Rango } \\
\text { Percentil }\end{array}$ \\
\hline 1 & 6 & 4 & 11 & 1 \\
\hline 5 & 10 & 6 & 17 & 5 \\
\hline 10 & 11 & 7 & 20 & 10 \\
\hline 15 & 12 & 8 & 21 & 15 \\
\hline 20 & 13 & 9 & 23 & 20 \\
\hline 25 & & & 24 & 25 \\
\hline 30 & 14 & 10 & 25 & 30 \\
\hline 35 & & & 26 & 35 \\
\hline 40 & 15 & 11 & 27 & 40 \\
\hline 45 & & & 28 & 45 \\
\hline 50 & 16 & 12 & 29 & 50 \\
\hline 55 & & & 30 & 55 \\
\hline 60 & 17 & 13 & 31 & 60 \\
\hline 65 & & 14 & 32 & 65 \\
\hline 70 & 18 & 15 & 33 & 70 \\
\hline 75 & & & 34 & 75 \\
\hline 80 & 19 & 16 & 35 & 80 \\
\hline 85 & 20 & 17 & 36 & 85 \\
\hline 90 & 21 & 18 & 38 & 90 \\
\hline 95 & 22 & 19 & 41 & 95 \\
\hline 99 & 25 & 24 & 49 & 99 \\
\hline $\mathbf{M}$ & 16.94 & 12.62 & 28.81 & $\mathbf{M}$ \\
\hline D.E. & 3.93 & 4.27 & 7.41 & D.E. \\
\hline $\mathrm{N}=942$ & & & & \\
\hline
\end{tabular}

